\title{
Studies of $B$ Hadron Rare Decays and Lifetimes at the TeVatron
}

\author{
M. D. Corcoran \\ for the CDF and D0 Collaborations \\ Rice University - Department of Physics and Astronomy \\ Houston, TX 77005 - USA
}

\begin{abstract}
The TeVatron collider at Fermilab is working well, and both the CDF and D0 experiments are collecting high-quality data at an impressive rate. The $B$ physics program at the TeVatron is producing a wide range of new results. In this talk [1] I summarize recent results for the search for the decays $B_{s}\left(B_{d}\right) \rightarrow \mu \mu$ and $B$ hadron lifetimes.
\end{abstract}

$1 \quad B_{s} \rightarrow \mu \mu$ and $B_{d} \rightarrow \mu \mu$

The decays $B_{s} \rightarrow \mu \mu$ and $B_{d} \rightarrow \mu \mu$ are flavor changing neutral current decays in the Standard Model and are therefore highly suppressed. The decay $B_{d} \rightarrow \mu \mu$ is suppressed even more by the ratio of the CKM matrix elements $V_{t d} / V_{t s}$. Figure 1 shows the relevant Standard Model Feynman diagrams for $B_{s} \rightarrow \mu \mu$. The Standard Model expectations for these modes are $B R\left(B_{s} \rightarrow \mu \mu\right)=3.42 \pm 0.54 \times 10^{-9}$ and $B R\left(B_{d} \rightarrow \mu \mu\right)=1.0 \pm$ $0.14 \times 10^{-10}[2]$, still far from the current experimental limits. But many models for physics beyond the Standard Model, particularly Supersymmetry, enhance these branching ratios by orders of magnitude. Therefore observation of $B_{s}\left(B_{d}\right) \rightarrow \mu \mu$ would be evidence for new physics.

The new D0 limit on this decay mode, based on about $2 \mathrm{fb}^{-1}$ data, is the first physics result from $\mathrm{D} 0$ to use their L0 silicon detector. L0, inserted during the spring 2006 shutdown, provides a substantial improvement in identification and measurement of displaced vertices.

The D0 search for $B_{s} \rightarrow \mu \mu$ requires two muons with $p_{t}>2.5 \mathrm{GeV}$ and $|\eta|<2$ which form a good vertex. A likelihood ratio (LHR) is formed

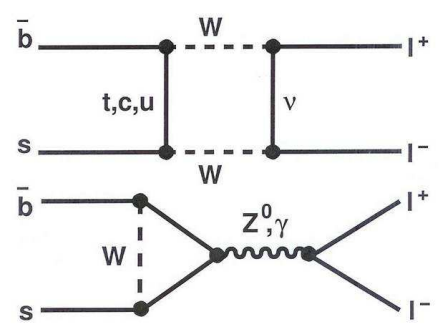

Figure 1: Standard Model diagrams for the decay $B_{s} \rightarrow \mu \mu$. using six variables: muon isolation, transverse decay length significance, $B$ impact parameter, minimum $\mu$ impact parameter, vertex $\chi^{2}$ probability, and pointing angle (the angle between the vector from the primary to the secondary vertices and the momentum of the reconstructed dimuon system). For each variable a probability density function (PDF) is determined for both signal (from Monte Carlo) and background (from $M_{\mu \mu}$ sidebands). The likelihood ratio for each event is then

$$
\mathcal{L}=\frac{\prod S_{i}}{\prod S_{i}+\Pi_{i}}
$$

where $S_{i}$ is the PDF for the signal and $B_{i}$ is the PDF for the background for variable $i$. If the variables do a good job of distinguishing signal from background, $\mathcal{L}$ is peaked near 1 for signal and near 0 for background. The left side of Figure 2 shows the LHR for signal Monte Carlo and background, with the cut indicated by the arrow. The right side of figure 2 shows the LHR vs. $M_{\mu \mu}$ plot for the RunIIa (pre-upgrade) dataset, with the final signal region in 

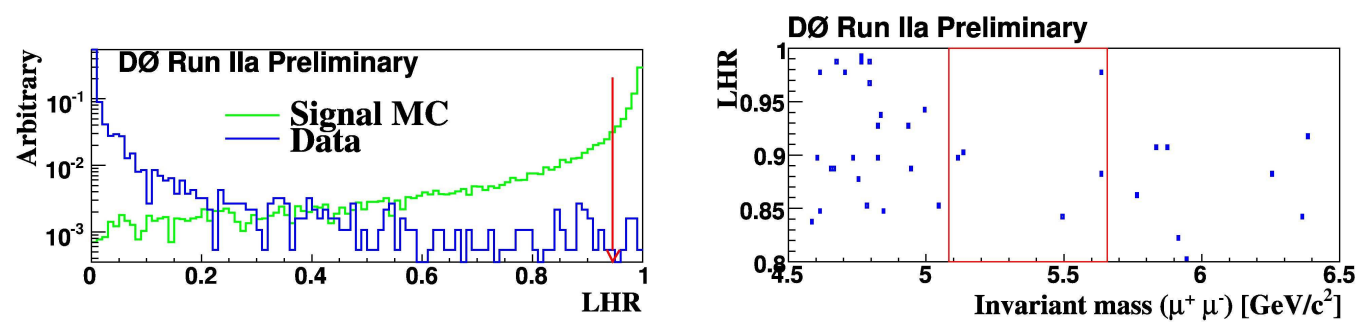

Figure 2: D0 result for the LHR for signal and background (left); the arrow indicates the value of the cut in LHR. The right plot is the LHR vs. $M_{\mu \mu}$ for the RunIIa (pre-upgrade) dataset.

dimuon mass indicated. In the RunIIa dataset, one event passes both LHR and mass cuts, while in the RunIIb dataset two events pass both cuts. The expected total background is 2.3 \pm 0.36 , so no excess of events over background is observed. The total flux of $B_{s}$ decays for this data sample is determined from the normalization mode $B^{+} \rightarrow J / \psi K^{+}$with $J / \psi \rightarrow \mu \mu$. The $95 \%$ CL limit is $B R\left(B_{s} \rightarrow \mu \mu\right)<9.3 \times 10^{-8}$, which is 27 times the Standard Model expectation. More information on this analysis can be found in the D0 conference note [3]

$\mathrm{CDF}$ has a recent result for both $B_{s}\left(B_{d}\right) \rightarrow \mu \mu$ based on $780 p b^{-1}$ of data. [4] Their data is divided into two subsets, one subset having both muons in their central muon detector $(\mathrm{CMU})$, and the other subset allowing one muon to be in their central muon extension (CMX). For the first subset, both muons must have $p_{t}>2 \mathrm{GeV}$ and $|\eta|<0.6$. For muons in the CMX, the requirements are $p_{t}>2.2 \mathrm{GeV}$ and $0.6<|\eta|<1.0$. The $p_{t}$ of the muon pair must be greater than $4 \mathrm{GeV}$, and they must form a good vertex. In this analysis, a likelihood ratio is formed from three variables: the $\mathrm{B}$ isolation, the $3 \mathrm{D}$ pointing angle, and the probability of $c \tau$, given the known $B_{s}\left(B_{d}\right)$ lifetime. Figure 3 left shows the LHR distributions for signal and background. Figure 3 right shows the final LHR vs. dimuon mass, with the signal regions indicated. For $B_{s}$ one event is observed with an expected background of $1.27 \pm 0.37$, and for $B_{d}$ two events are observed with an expected background of $2.45 \pm 0.40$. Again there is no excess over the expected background. CDF has also used the normalization mode $B^{+} \rightarrow J / \psi K^{+}$with $J / \psi \rightarrow \mu \mu$. The resulting limits at 95\% CL are $B R\left(B_{s} \rightarrow \mu \mu\right)<1.0 \times 10^{-7}$ and $B R\left(B_{d} \rightarrow \mu \mu\right)<2.3 \times 10^{-8}$ which are 20 and 230 times the Standard Model expectation, respectively.

We can (unofficially) combine the CDF and D0 limits to obtain a combined 95\% CL limit $B R\left(B_{s} \rightarrow \mu \mu\right)<5.8 \times 10^{-8}$, about 17 times the SM expectation. Both experiments have substantially more data, so this limit can be expected to improve in the near future.

\section{B Hadron Lifetime Measurements}

Lifetimes for B hadrons can be calculated in Heavy Quark Expansion models[5], so precision measurements of lifetimes are good tests of this model. D0 has recent measurements on $\Lambda_{b}$, both through the exclusive channel $\Lambda_{b} \rightarrow J / \Psi \Lambda$ with $J / \psi \rightarrow \mu \mu$, and through the semileptonic channel $\Lambda_{b} \rightarrow \Lambda_{c} \mu \nu X$ with $\Lambda_{c} \rightarrow K_{s} p$. CDF has a measurement in the exclusive channel only.

Figure 4 shows the CDF result [6] for the $\Lambda_{b}$ mass and $c \tau$ distribution in the exclusive mode mentioned above. There are 532 candidate events, and a maximum likelihood fit on 

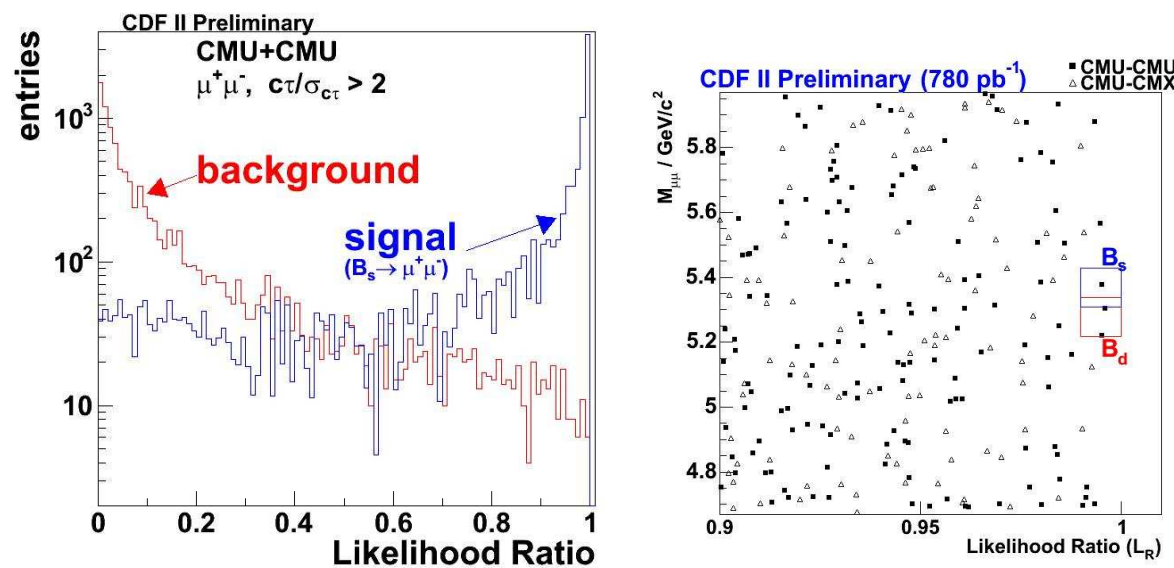

Figure 3: CDF result for the likelihood ratio for signal and background (left) and likelihood ratio vs. $M_{\mu \mu}$ (right) for the data.
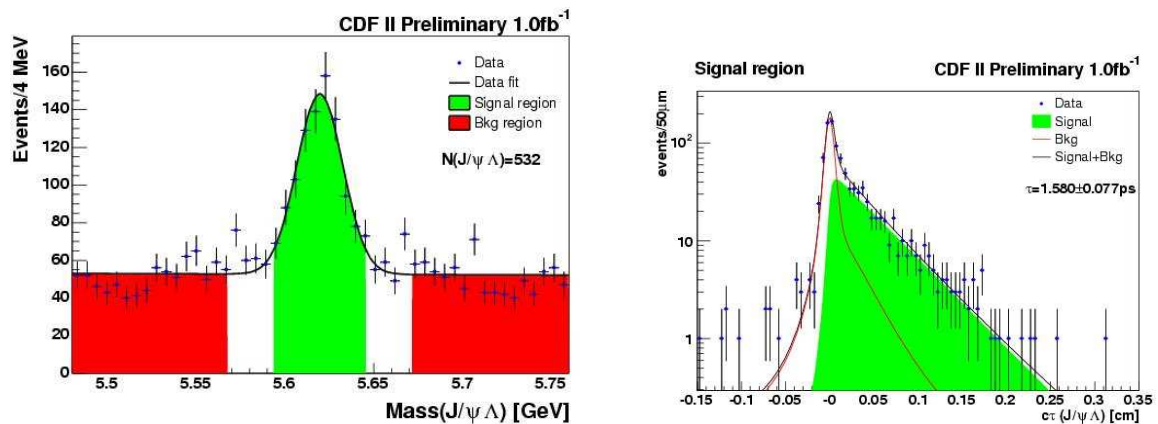

Figure 4: CDF results: $\Lambda_{b}$ mass distribution for fully-reconstructed decays (left) and the $c \tau$ distribution for $\Lambda_{b}$ candidates.

three variables (mass, $c \tau$ and $c \tau$ error) yields the lifetime measurement shown in figure 5 .

The first D0 measurement of the $\Lambda_{b}$ lifetime is based on $172 \pm 21$ events in the same exclusive mode. D0 has also measured the $\Lambda_{b}$ lifetime in the semileptonic mode, which has much higher statistics but also much higher background. [7] The $\Lambda_{b}$ lifetime from the semileptonic mode yields $4437 \pm 329$ events. Figure 5 shows the world data on the $\Lambda_{b}$ lifetime. There is about a 2 standard deviation discrepancy on the $\Lambda_{b}$ lifetime between CDF and D0. Both experiments have significantly more data, so one would expect this discrepency to be resolved soon.

$\mathrm{CDF}$ has also made precision measurements for $B^{+} \rightarrow J / \psi K^{+}, B^{0} \rightarrow J / \psi K^{*}$ or $J / \psi K^{*}$, and $B_{s} \rightarrow J / \psi \Phi$. All are in good agreement with previous measurements and with expectations. Figure 5 right shows the world data on the ratio of lifetimes $\tau\left(B^{+}\right) / \tau\left(B^{0}\right)$.

More information on all these measurements is available on the excellent websites of both 
experiments [8].
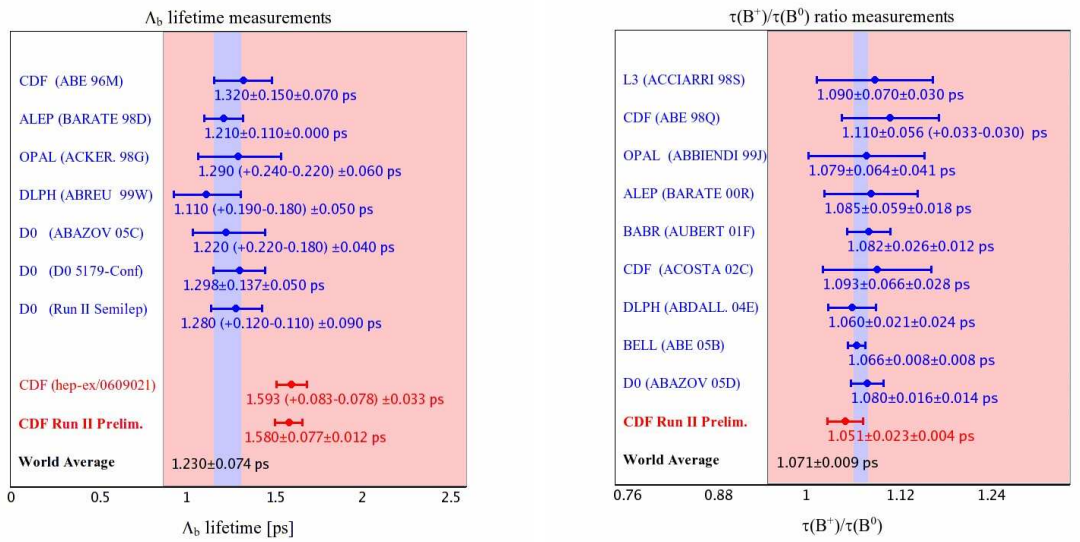

Figure 5: World results for the $\Lambda_{b}$ lifetime (left) and for the $\tau\left(B^{+}\right) / \tau\left(B^{0}\right)$ (right).

\section{References}

[1] http://indico. cern. ch/contributionDisplay $\cdot$ py? contribId=203\&sessionId=5\& conf Id=9499

[2] A. J. Buras, Phys. Lett. B566, 115 (2003).

[3] D0 conference note D05344-CONF, available from the website noted below.

[4] CDF public note 8176, available from the website noted below.

[5] F. Gabbiani, Phys. Rev. D68 114006 (2003).

[6] CDF public note 8524 .

[7] Fermilab-Pub-07/094-E (submitted to Phys. Rev. Lett.)

Fermilab-Pub-07/205-E (submitted to Phys. Rev. Lett.)

[8] http://www-cdf.fnal.gov/physics/new/bottom/bottom.html http://www-d0.fnal.gov/Run2Physics/WWW/results.b.htm 